ÉGYPTE monde arabe

\section{Égypte/Monde arabe}

\section{$21 \mid 1995$}

Économie égyptienne et perspectives de paix au Proche-Orient

\title{
La captation de la rente arabe par l'Égypte
}

\section{Marie-France Vernier}

\section{(2) OpenEdition}

Journals

Édition électronique

URL : https://journals.openedition.org/ema/370

DOI : 10.4000/ema.370

ISSN : 2090-7273

Éditeur

CEDEJ - Centre d'études et de documentation économiques juridiques et sociales

Édition imprimée

Date de publication : 31 mars 1995

Pagination : 19-30

ISSN : 1110-5097

\section{Référence électronique}

Marie-France Vernier, «La captation de la rente arabe par l'Égypte », Égypte/Monde arabe [En ligne], 21 | 1995, mis en ligne le 08 juillet 2008, consulté le 07 juillet 2022. URL : http://journals.openedition.org/ ema/370 ; DOI : https://doi.org/10.4000/ema.370

Ce document a été généré automatiquement le 7 juillet 2022.

Tous droits réservés 


\title{
La captation de la rente arabe par l'Égypte
}

\author{
Marie-France Vernier
}

Du début des années 70 au milieu des années 80, l'Égypte a réussi à s'approprier des rentes liées à la rente pétrolière circulant dans le monde arabe ou puisées dans d'autres régions du monde ${ }^{1}$. Malgré la diminution récente de ces revenus, l'attitude rentière continue de caractériser la politique économique égyptienne, sur les plans extérieur et intérieur. Or, le règlement en cours du conflit israélo-arabe peut modifier les conditions de cette appropriation. Dans un premier temps, nous considérerons la position de l'Égypte dans la région. La dynamique de son économie repose, en partie au moins, sur la perception de devises en provenance du monde arabe et des pays occidentaux. Moins commerciales que financières, les relations égypto-arabes, dominées par les aides financières et les revenus des travailleurs émigrés, révèlent la faible intégration économique du Moyen-Orient et son hétérogénéité. Après avoir montré comment s'articulent les deux notions de paix et de développement, nous aborderons les conséquences de la paix des points de vue égyptien et arabe. La libéralisation économique en cours en Égypte peut en effet bénéficier de la fin du conflit, une accélération conséquente des échanges commerciaux et financiers dans la région étant susceptible d'alléger la contrainte extérieure. La fin de l'état de belligérance entre Israël et ses voisins, en favorisant une stabilisation politique de la région, permettra aux États de penser puis d'établir des relations entre eux dans une optique de long terme. Il s'agira alors d'encourager une intégration économique et financière.

\section{L'Égypte et le Moyen-Orient}

\section{La position de l'Égypte dans le monde arabe}

2 L'économie égyptienne se caractérise par sa dépendance à l'égard de flux financiers en provenance de l'étranger. Ce sont, principalement, les recettes liées au transit par le 
canal de Suez et à l'activité touristique, les transferts des travailleurs émigrés et l'aide étrangère, revenus produits par la circulation de la rente pétrolière d'une région à l'autre du Moyen-Orient et par certaines situations de monopole.

Les recettes que perçoivent les producteurs de pétrole sont une rente au sens où l'entend D. Ricardo ${ }^{2}$ : le pétrole est une ressource rare, non reproductible, de productivité variable selon les gisements, le propriétaire du site le plus fertile bénéficiant des coûts de production les moins élevés. Cependant, le prix de vente sur le marché est déterminé par les coûts en vigueur sur les sites les moins fertiles. Le propriétaire percevra une rente dite différentielle, du fait de la différence existant entre ses propres coûts et le prix du marché. Au niveau régional, l'ensemble des recettes perçues par les pays producteurs de pétrole constitue une rente. Depuis le contre-choc pétrolier de 1966, le prix du baril a chuté, entraînant une diminution de la rente perçue par les producteurs.

\section{Les rentes perçues par l'Égypte}

4 Nous distinguerons parmi celles-ci les revenus liés à la rente pétrolière arabe et ceux découlant de situations de monopole. La rente pétrolière est captée par le biais du trafic pétrolier sur le canal de Suez, des migrations des travailleurs égyptiens et de l'aide arabe. La première marchandise circulant sur le canal de Suez est en effet le pétrole ${ }^{3}$ en provenance des pays arabes et de l'Iran, dans le sens sud-nord. Les recettes perçues à cette occasion par l'Autorité du canal peuvent être considérées comme une redistribution d'une partie de la rente pétrolière. Les compagnies, propriétaires du pétrole acquis par les producteurs, jouent le rôle d'intermédiaires. En 1992, le transport du pétrole d'origine saoudienne représentait $41 \%$ du tonnage pétrolier total dans le sens sud-nord, suivi par l'Iran (21\%) et les Emirats arabes unis $(8 \%)^{4}$.

5 Quant à l'émigration égyptienne, elle résulte des besoins en main-d'œuvre des pays arabes producteurs de pétrole. Les transferts ainsi perçus par l'Égypte sont substantiels, puisqu'elle arrive en tête des pays arabes récepteurs de transferts des émigrés tout au long des années 80. Par ailleurs, le retour des Égyptiens dans leur pays à la suite de la guerre du Golfe et l'élévation des taux d'intérêt en Égypte ont entraîné une augmentation des transferts des émigrés. En raison de son attitude durant le conflit, l'Égypte a pu en outre bénéficier du soutien des monarchies du Golfe, qui emploient ses ressortissants d'autant plus volontiers. Enfin, la nouvelle politique économique a réorienté les transferts vers les circuits officiels (tableau 1).

6 L'aide dont Le Caire bénéficie est surtout d'origine occidentale et, dans une moindre mesure, arabe (tableau 2). Ayant boycotté le signataire des accords de Camp David, les pays arabes avaient pratiquement suspendu leur aide entre 1979 et 1985. L'amélioration des relations égypto-arabes est devenue évidente après la guerre du Golfe: les pays arabes alliés contre l'Irak ont alors soutenu l'Égypte tant par des dons que par des remises de dettes.

7 Le canal de Suez et le tourisme constituent en revanche des cas de monopole. Le premier, voie de passage la plus rapide pour le transport des marchandises entre la mer Rouge et la Méditerranée, a rapporté, pour l'année fiscale 1991/92, près de deux milliards de dollars. L'activité touristique relève du même cas de figure. L'Égypte, qui possède sur son territoire les ruines d'une civilisation ancienne et reconnue, détient le monopole de la propriété et de l'exploitation de ces sites. Ce "secteur économique 
repose dans une certaine mesure sur le tourisme arabe puisqu'on moyenne, depuis $1987 / 88$, ce dernier représente près de $40 \%$ des nuitées et des visiteurs 5 . Cette activité étant, par ailleurs, très dépendante de l'actualité politique, les revenus peuvent varier amplement d'une année à l'autre (cf. tableau 1).

8 L'aide étrangère, reçue par Le Caire peut être considérée comme une rente politique liée à une situation de monopole. En effet, l'économie égyptienne est une des rares à bénéficier d'un tel soutien, dans le monde arabe en particulier. Parmi les facteurs qui expliquent de ce privilège, nous pouvons évoquer sa situation géographique, la taille de sa population et sa nécessaire stabilité politique. Les nations occidentales ont été particulièrement généreuses en lui permettant d'accumuler une dette considérable jusqu'en 1991. Après la guerre du Golfe, les créanciers du Club de Paris ont accepté de diminuer de $50 \%$ la valeur actualisée nette de la dette rééchelonnable, et de rééchelonner la moitié restante sur 20 à 25 ans. .

\section{Les relations égypto-arabes}

9 Les relations que l'Égypte entretient avec le Moyen-Orient présentent un double aspect. D'une part, elles manifestent une meilleure intégration à l'économie mondiale qu'à l'économie moyen-orientale. D'autre part, la faiblesse des flux commerciaux contraste avec l'ampleur des flux financiers au sein de la région. Les échanges de biens s'effectuent principalement avec les États-Unis, l'Union européenne et le Japon ${ }^{6}$. Comme c'est le cas pour l'ensemble des pays arabes et ceux en voie de développement, les exportations et les importations égyptiennes concernent surtout les économies du Nord. Ainsi, en 1990, la part des pays arabes dans le commerce extérieur de l'Égypte ne représentait que $5,5 \%$ de ses exportations et $6 \%$ de ses importations ${ }^{7}$.

La faiblesse structurelle de la balance des paiements égyptienne réside dans l'insuffisance des ressources dont elle dispose compte tenu dé la consommation interne ${ }^{8}$. Ainsi, les échanges commerciaux sont peu développés alors que les services et les transferts ne permettent pas de rééquilibrer la balance des transactions courantes. De plus, le pétrole constitue un poste important des exportations égyptiennes, $50 \%$ en 1990/91, après l'élévation des prix du pétrole ${ }^{9}$. Or, en raison de la sensibilité de ces exportations aux événements internationaux, les résultats du commerce extérieur sont fluctuants.

\section{L'importance des remises et de l'aide arabe}

11 Parmi les apports invisibles en provenance des pays arabes, il en est de deux sortes : les aides gouvernementales et les revenus envoyés par les Égyptiens émigrés en pays arabes. Aussi importants ces revenus soient-ils, les aides gouvernementales sont une constante de la balance des paiements. Le soutien des pays arabes a été relativement modeste jusqu'à la fin des années 80 , mais les flux financiers en provenance de ces derniers sont généralement supérieurs aux échanges commerciaux inter-arabes.

Depuis le milieu des années 70, l'Égypte est le premier bénéficiaire arabe des envois des travailleurs émigrés dans cette région, suivie ensuite par le Maroc et le Yémen du Nord. De 1979 à 1989, les Égyptiens ont envoyé en moyenne chaque année près de 3,229 milliards de dollars contre 2,285 milliards de dollars entre 1973 et 1978 (tableau 3). 
13 Les flux d'aide en provenance des pays arabes ont été plus irréguliers en raison, notamment, de leur boycott de l'Égypte. Si, pendant la deuxième période, elle a reçu en moyenne par an 23 millions de dollars, durant la période précédente. elle en a perçu plus d'1,5 milliard, suivie ensuite par la Syrie et la Jordanie avec un peu plus de 500 millions de dollars (tableau 4). Cependant, elle bénéficie, depuis la guerre du Golfe, d'un soutien plus large, et la nouvelle politique économique conseillée par les institutions financières internationales, en permettant la stabilisation de la livre égyptienne, favorise le retour des capitaux égyptiens.

\section{La faible intégration régionale}

14 La réflexion de John Waterbury (1978) selon laquelle l'unité arabe et/ou l'intégration économique est une nécessité pour l'Égypte et non un choix politique. est toujours d'actualité.

15 Les flux financiers circulant au sein de la région nourrissent l'illusion d'une intégration financière où les pays du Golfe distribueraient une partie de leur richesse vers les pays arabes plus pauvres. En réalité, les nombreuses tentatives d'intégration régionale n'ont pas abouti ${ }^{10}$. Très dépendantes des aléas politiques, les relations inter-régionales sont fragiles. Si intégration il y a, elle semble reposer sur les mouvements d'hommes et de capitaux. Ceux-ci se réalisent plutôt au sein de la région, alors que les flux de marchandises sont tournés vers l'extérieur. Un déséquilibre apparaît donc entre la tendance des flux humains et financiers et celle des flux commerciaux. L'économie arabe est fragmentée parce que chaque pays est davantage intégré au marché mondial qu'à la région à laquelle il appartient.

\section{Une région très hétérogène}

16 Le monde arabe se caractérise par l'hétérogénéité des économies qui le composent. Les riches pays détenteurs de l'or noir côtoient aussi bien des États fournisseurs de maind'œuvre comme l'Égypte, la Jordanie et le Yémen du Nord, que des économies exportatrices de produits manufacturés comme Israël, la Turquie et la Jordanie ${ }^{11}$.

L'Arabie Saoudite, l'Irak, le Koweït, les Emirats arabes unis et, dans une moindre mesure, la Libye sont détenteurs des réserves pétrolières les plus importantes au monde et bénéficient de très faibles coûts de production. Au niveau de chaque pays, en particulier les monarchies du Golfe, l'État occupe un rôle central puisqu'il reçoit et distribue la rente pétrolière. Nous sommes dans le cas d'Etals rentiers où le revenu disponible par habitant est souvent très élevé alors que très peu participent à la génération de la rente. Leurs dépenses militaires sont considérables. Parmi les pays en voie de développement, le Moyen-Orient est la région ayant engagé le plus de fonds dans ce domaine. Ces économies sont ainsi extrêmement dépendantes de la manne pétrolière. Depuis le début des années 70 , les taux de croissance du PIB et de la production de pétrole suivent les mêmes évolutions ${ }^{12}$.

\section{La faiblesse des échanges intra-arabes}

18 En matière d'aide, les donateurs les plus généreux sont l'Arabie Saoudite et le Koweït. Les États bénéficiaires dépendent donc, à leur tour, de la rente pétrolière circulant dans 
la région. En 1989, l'aide officielle nette versée par les pays et organisations arabes aux pays arabes a atteint 823 millions de dollars ${ }^{13}$. La même année, les transferts des travailleurs émigrés en provenance de ces pays s'élevaient à 7,539 milliards de dollars ${ }^{14}$. Par contre, les échanges commerciaux dans la région sont faibles, à l'image de ceux de l'Égypte (tableau 5 p. suivante). Ainsi, en 1991, la part des exportations et des importations inter-arabes dans l'ensemble du commerce extérieur arabe ont été respectivement de $8 \%$ et $9 \%{ }^{15}$. L'Arabie Saoudite est la plus dynamique dans ce domaine : elle réalise $45 \%$ des exportations. Quant aux importations, le Bahrein, les Emirats arabes unis et l'Arabie Saoudite en ont réalisé respectivement $17.4 \%, 11,4 \%$ et $10,8 \%{ }^{16}$.

\section{De la guerre a la paix}

Le règlement du conflit israélo-arabe n'en est qu'à ses débuts, notamment en ce qui concerne les négociations israélo-palestiniennes. Eu égard à ce contexte, notre analyse s'articulera autour de trois axes : la relation qu'entretiennent les notions de paix et de développement économique, les attentes de l'Égypte et les chances d'une intégration économique plus importante.

\section{Paix et développement}

21 Le lien paix/développement est complexe. Le règlement du conflit avec Israël devrait en principe conduire au désarmement de la région et selon une attente légitime, la pacification des relations régionales devrait favoriser le développement au bénéfice de tous. Jusqu'à présent, l'état de guerre ne s'est pas traduit par une croissance économique équitable.

Cela dit, les notions de désarmement et de développement ne sont pas nécessairement liées dans la pensée économique ${ }^{17}$. La défense de la nation stimule en effet ('emploi et de multiples activités économiques. Les mercantilistes revendiquent ainsi un État "économiquement et militairement fort, unifié autour du prince»" De plus, en entretenant le sentiment national, l'état de guerre renforce l'esprit d'entreprise nécessaire à l'établissement d'une économie puissante. L'« union autour du prince » peut qualifier la situation des États rentiers du monde arabe, qui entretiennent leur influence régionale en accordant des aides. Ainsi, alors que l'intégration économique est faible, la coopération politique et militaire est considérable. Cependant, les dépenses militaires constituent un prélèvement sur les ressources disponibles alors que les besoins en infrastructures et en produits agricoles et industriels sont considérables dans les pays en voie de développement. Et le passage à une situation de paix n'entraînera pas immédiatement la conversion des activités militaires en activités civiles. 


\section{Les attentes de l'Égypte en cas de paix}

\section{Les rentes}

Parmi les rentes de l'Égypte, le transit par le canal de Suez, les transferts des travailleurs émigrés et l'aide arabe sont les revenus les plus sensibles à la conjoncture pétrolière et politique de la région.

L'activité du canal peut diminuer avec la réouverture des oléoducs qui avaient été fermés à cause du conflit. Quant au niveau élevé des transferts privés, il ne saurait durer puisqu'il est lié à la politique des taux d'intérêts élevés actuellement pratiquée en Égypte et à la faveur accordée à ses ressortissants par les pays du Golfe. D'autant que ceux-ci vont devoir tôt ou tard affronter la concurrence des travailleurs palestiniens.

Si l'attitude du Caire pendant la guerre du Golfe a permis une relance de l'aide arabe (cf., plus haut, le tableau 2), la fin du conflit avec Israël et les demandes de financement des Territoires occupés peuvent conduire à une réévaluation, à la baisse, de l'aide accordée à l'Égypte.

\section{La promotion des exportations}

Les attentes de l'Égypte se justifient en partie par le déficit de ses échanges commerciaux. Aussi, outre la politique d'ajustement qui vise à le réduire. la promotion des exportations pourrait alléger la contrainte pesant sur la balance des paiements. De nouvelles relations avec les économies palestinienne et israélienne ainsi qu'une plus grande ouverture sur le monde arabe jouent alors un rôle clef.

Bien qu'elle soit en paix avec Israël depuis la signature des accords de Camp David, l'Égypte entretient très peu d'échanges commerciaux avec ce pays. La fin du conflit permettra à Israël d'entamer avec la région des relations dont l'Égypte pourrait bénéficier. De plus, ayant une frontière commune avec la Palestine, elle peut établir des relations commerciales et financières avec les territoires autonomes, ^d'autant qu'il s'agira d'une économie en pleine reconstruction,

28 Les accords de Camp David avaient fait perdre au Caire, du moins officiellement, le soutien financier des pays arabes. La pacification des relations, israélo-arabes va améliorer sa position au niveau régional. La politique d'ajustement structurel de l'économie égyptienne est sévère : une stimulation du commerce extérieur par une plus grande ouverture sur le monde arabe serait donc la bienvenue. Les autres marchés Europe, États-Unis - sont difficiles à conquérir. La politique commerciale égyptienne doit donc être conçue au niveau régional.

\section{Stimulation de l'intégration économique du monde arabe ?}

La fin du conflit entre Israël et ses voisins va également modifier les relations interarabes elles-mêmes, changement qui, s'il est avant tout d'ordre politique, peut influencer l'économie des pays concernés. Les anticipations des différents acteurs de la scène arabe sont essentielles. La cessation d'une guerre vieille de près d'un demi-siècle permet-elle d'espérer une véritable stabilité politique? La violence des conflits a marqué les populations arabes et leurs dirigeants: le développement des relations israélo-arabes prendra du temps. 
30 L'économie palestinienne a un besoin énorme en capital. La mise en place d'infrastructures peut être démarrée avec des entreprises arabes. L'économie palestinienne a besoin d'une coopération régionale que les gouvernements peuvent favoriser par des politiques économiques stimulant les relations commerciales et financières. De plus, la dépendance de l'économie palestinienne vis-à-vis de l'économie israélienne dans le commerce et l'emploi est profonde: ce peut être là un axe de développement des relations israélo-arabes.

Il semble difficile que l'intégration économique s'accélère avant la création d'une zone de libre échange comme l'Union européenne et l'ALENA ${ }^{19}$. Dans le cas européen, maigre la similitude des niveaux de développement des pays concernés et le volume des échanges intra-européens, l'union est un processus lent et difficile qui, entamé en 1951 par l'union douanière pour le charbon et les produits sidérurgiques (CECA), n'est pas encore achevé.

Pour les pays producteurs de pétrole, l'ère pétrolière n'est que temporaire. La communauté d'intérêts autour de cette incertitude devrait stimuler des efforts, conçus régionalement, en laveur des activités productives. Les pays fournisseurs de maind'œuvre et récepteurs de l'aide arabe sont aussi concernés par la diminution de la rente pétrolière.

Les bouleversements régionaux en cours sont considérables. L'économie égyptienne et l'ensemble du monde arabe bénéficieraient d'un approfondissement des relations commerciales régionales. Mais les exigences de l'économie ne sont pas suffisantes compte tenu de l'influence déterminante des facteurs politiques. De plus, la création de zones de libre-échange, en Europe et en Amérique du Nord, montre qu'actuellement, une région est d'autant plus dynamique que ses composantes sont proches les unes des autres. La paix n'est peut être pas une, condition suffisante à la création d'une véritable intégration.

\section{ANNEXES}

Tableau 1 : Les rentes de l'économie égyptienne (en millions de livres égyptiennes)

\begin{tabular}{|l|l|l|l|l|l|l|}
\hline & $1987 / 88$ & $1988 / 89$ & $1989 / 90$ & $1990 / 91$ & $1991 / 92$ & $1992 / 93$ \\
\hline Canal de Suez & 868 & 1.437 & 1.619 & 3.995 & 6.463 & 6.473 \\
\hline Tourisme & 1.922 & 2.304 & 2.762 & 2.687 & 5.699 & 5.897 \\
\hline Remises des trav. émigrés & 7.573 & 9.196 & 9.764 & 11.204 & 18.197 & 24.193 \\
\hline Transfets officiels & 1.545 & 1.854 & 2.527 & 4.058 & 3.453 & 4254 \\
\hline Total & 11.928 & 14.792 & 16.672 & 21.944 & 33.813 & 40.817 \\
\hline
\end{tabular}




\begin{tabular}{|l|l|l|l|l|l|l|}
\hline PIB & 58.700 & 65.600 & 81.300 & 103.300 & 131.057 & n.d. \\
\hline Ratio rentes/PIB & $36 \%$ & $40 \%$ & $36 \%$ & $35 \%$ & $44 \%$ & \\
\hline
\end{tabular}

Source : Banque centrale.

Tableau 2 : Aide officielle reçue par l'Égypte (en millions de \$ courants)

\begin{tabular}{|l|l|l|l|l|l|l|}
\hline & 1985 & 1986 & 1987 & 1988 & 1989 & 1990 \\
\hline Aide totale & 1.791 & 1.716 & 1.773 & 1.537 & 1.568 & 5.604 \\
\hline Aide arabe bilatérale & 25 & 53 & 74 & 17 & 15 & 2.324 \\
\hline Aide arabe totale & -27 & 54 & 65 & 8 & 83 & \\
\hline
\end{tabular}

Sources : Banque mondiale, World Developpent Report, Oxford University Press, NewYork, 1992,308 p., Rapport arabe uni, 1992, Van den Boogaerde P., 1991.

Tableau 3 : Remises des travailleurs émigrés perçues par l'Égypte, la Jordanie, le Maroc et le Yémen du Nord (remises moyennes annuelles, en millions de \$ courants)

\begin{tabular}{|l|l|l|l|}
\hline & $1973-1989$ & $1973-1978$ & $1979-1989$ \\
\hline Égypte & $2.285,15$ & 724,02 & $3.229,03$ \\
\hline Jordanie & 596,60 & 259,35 & 806,54 \\
\hline Maroc & 861,17 & 458,33 & $1.101,00$ \\
\hline Yémen du Nord & 677,31 & 582,12 & 703.30 \\
\hline
\end{tabular}

Source : Van den Boogaerde P., 1991.

Tableau 4 : Aide officielle nette déboursée par les pays et les organisations arabes en faveur de la Syrie, de l'Égypte et de la Jordanie (aide moyenne annuelle, en millions de \$ courants)

\begin{tabular}{|l|l|l|l|}
\hline & $1973-1989$ & $1973-1978$ & $1979-1989$ \\
\hline Syrie & 724,54 & 584,98 & 800,65 \\
\hline Égypte & 550,85 & $1.517,82$ & 23,41 \\
\hline Jordanie & 521,77 & 274,45 & 656,67 \\
\hline
\end{tabular}

Source : Van den Boogaerde P., 1991. 
Tableau 5 : Commerce extérieur arabe (en millions de \$ courants)

\begin{tabular}{|l|l|l|l|l|l|l|l|}
\hline & 1985 & 1986 & 1987 & 1988 & 1989 & 1990 & 1991 \\
\hline Exportations arabes totales & 107.226 & 78.933 & 92.672 & 91.526 & 112.359 & 140.552 & 123.423 \\
\hline$\cdot$ vers les pays arabes & 7.450 & 6.729 & 7.281 & 8.180 & 10.201 & 11.633 & 9.664 \\
\hline$\cdot$ vers l'Égypte & 350 & 180 & 118 & 202 & 261 & 256 & 459 \\
\hline Importations arabes totales & 93.955 & 85.214 & 82.223 & 92.888 & 94.846 & 104.006 & 109.463 \\
\hline$\cdot$ en provenance des pays arabes & 8.144 & 6.547 & 7.190 & 8.087 & 9.360 & $10.060 '$ & 97.349 \\
\hline * en provenance d'Egypte & 127 & 321 & 21 & 189 & 130 & 191 & 282 \\
\hline
\end{tabular}

Source : Fonds monétaire arabe, 1992.

Tableau 6 : Commerce extérieur égyptien (en millions de \$ courants)

\begin{tabular}{|l|l|l|l|l|l|l|l|}
\hline & 1985 & 1986 & 1987 & 1988 & 1989 & 1990 & 1991 \\
\hline \hline Exportations totales de l'Égypte & $1.838,1$ & $2.248,7$ & $2.037,2$ & $2.120,3$ & $2.647,7$ & $2.568,8$ & $3.838,2$ \\
\hline Importations totales de l'Égypte & $5.495,4$ & $8.787,8$ & $7.594,6$ & $8.657,2$ & $7.447,5$ & $9.170,0$ & $8.226,7$ \\
\hline
\end{tabular}

Source : Fonds monétaire arabe, 1992.

\section{NOTES}

1. Cet article reprend la problématique d'une thèse en cours sur le thème de L'économie rentière: approches dans le cas égyptien, sous la direction du professeur Pierre Salama.

2. Ricardo D., Des principes de l'économie politique et de l'impôt, Science Flammarion, Paris, 1971, 379

p.

3. En 1992, le pétrole représentait $46 \%$ des marchandises transportées à destination du Nord, et près du tiers des marchandises dans les deux sens. Revue du canal de Suez, 1992.

4. Revue du canal de Suez, op. cit.

5. Van Den BoogaerdeP., Financial Assistance from Arab Countries and Arab Regional Institution, Fonds monétaire international, Occasional papers $\mathrm{n}^{\circ}$ 87, Washington D-C, 1991, $102 \mathrm{p}$.

6. Banque centrale, Rapport annuel, divers numéros.

7. Banque centrale, Rapport annuel, 1992/93.

8. Voir Fonds monétaire arabe, Foreign Trade of Arab Countries 1991-199, Abu Dhabi, 1992, 238 p.

9. Vernier M.-F., La dollarisation, mémoire de DEA, Université Paris-Nord, 1991, 117 p.

10. Fond monétaire arabe, 1992.

11. Handoussa H. et Shafik N., "The Economy of Peace : the Egyptian Case ", Conférence sur L'Économie de la Paix au Moyen-Orient, Université de Harvard. novembre 1991,48 p. 
12. Richards A. et Waterbury J., Political Economy of the Middle East, American University of Cairo Press, Le Caire, 1990, $495 \mathrm{p}$.

13. Kousnetzoff N., Levet V. et Peltrault F., «L'après-pétrole dans les pays du Golfe ", Économie prospective internationale $\mathrm{n}^{\circ}$ 53, 1er trimestre 1993, La Documentation française, pp. 33 à 54.

14. Van Den Boogaerde P., op. cit.

15. Van Den Boogaerde P., id.

16. Fonds monétaire arabe, 1992.

17. Rapport arabe économique uni, 1992, $354 \mathrm{p}$.

18. Fontanel J. (sous la dir.), Économistes de la Paix, coll. Économie en plus, Presses universitaires de Grenoble, 1993, 82 p.

19. Zone de libre-échange entre les États-Unis, le Mexique et le Canada.

INDEX

Mots-clés : économie, monde arabe, rente

\section{AUTEUR}

MARIE-FRANCE VERNIER

CEDEJ 